\title{
Neural Fashion Image Captioning : Accounting for Data Diversity
}

\author{
Gilles Hacheme ${ }^{1}$ and Noureini Sayouti ${ }^{2}$ \\ ${ }^{1,2} \mathrm{Ai} 4$ Innov \\ ${ }^{2}$ Aix-Marseille University (Aix-Marseille School of Economics), \\ CNRS \& EHESS, Marseille, France \\ \{gilles.hacheme,noureini.sayouti\}@ai4innov.com
}

June 2021

\begin{abstract}
Image captioning has increasingly large domains of application, and fashion is not an exception. Having automatic item descriptions is of great interest for fashion web platforms hosting sometimes hundreds of thousands of images. This paper is one of the first tackling image captioning for fashion images. To contribute addressing dataset diversity issues, we introduced the InFashAIv1 dataset containing almost 16.000 African fashion item images with their titles, prices and general descriptions. We also used the well known DeepFashion dataset in addition to InFashAIv1. Captions are generated using the Show and Tell model made of CNN encoder and RNN Decoder. We showed that jointly training the model on both datasets improves captions quality for African style fashion images, suggesting a transfer learning from Western style data. The InFashAIv1 dataset is released on Github to encourage works with more diversity inclusion.
\end{abstract}

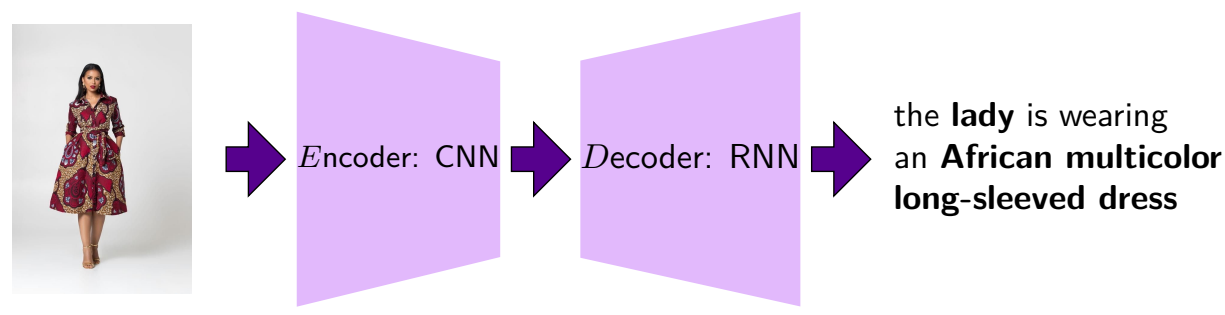

\section{Introduction}

Image captioning is the process aiming to associate a text description to an image in an automatic manner. In this process, a computer is trained to understand the visual content of an image and produce a corresponding descriptive 
sentence, the caption. This process combines two sub-fields of computer science namely: Natural Language Processing (NLP) and Computer Vision (CV). Automatic caption generation from a given image could have several use cases like recommendations in editing applications, virtual assistants, image indexing, assisting visually impaired persons to understand the content of an image (Srinivasan and Sreekanthan, 2018). Image captioning is a challenging task and it recently drew lots of attention from researchers in CV (Vinyals et al., 2015; Wang et al., 2020; Yang et al., 2020).

Most of research works on image captioning focus on broad areas with images of different types showcasing humans in their daily life or objects used by them on a daily basis (Everingham et al., 2009; Lin et al., 2014b). However, one of the areas in which image captioning has been used very little is the field of fashion, despite all its attractiveness for researchers in AI and more specifically in CV. Indeed, in the last decade the fashion industry attracted many researchers in CV. One reason could be its rapid growth and its huge estimated value. Indeed, according to Statista, the global apparel market will grow from $\$ 1.5$ trillion in 2020 to 2.25 trillion by 2025 . Yet, to the best of our knowledge, very few papers have applied image captioning to the fashion industry.

The work by Yang et al. (2020) is one of the pioneering work applying image captioning to fashion images. The captions generated by Yang et al. (2020) in their paper have both an objective part (description of attributes) and a subjective part aiming to embellish outfits descriptions to attract customers' attention and induce them to buy. The usefulness of this type of descriptions are very marketing oriented. In this paper, we will limit ourselves to generating objective descriptions based on outfits' attributes. Objective description generation is useful to automate attribute extraction from fashion images. This could be helpful to perform a bunch of tasks. Indeed, despite its direct potential usefulness for item description, categorization and recommendation on fashion web sites, it could be used as building block for more advanced tasks such as image generation from text (Zhu et al., 2017). Indeed, for this kind of tasks objective descriptions are needed, and the ability to generate such descriptions automatically is very useful as it will allow to easily build huge databases to feed this kind of algorithm. Objective descriptions can also be used in any fashion web platform or market place to automate image descriptions and classification according to detected attributes.

In this paper we are using the model proposed by Vinyals et al. (2015) named Show and tell, based on encoder-decoder architecture in which the encoder is a Convolutional Neural Network $(\mathrm{CNN})$ and the decoder is a Recurrent Neural Network (RNN). They initialized the encoder using a pretrained CNN on the ImageNet dataset (Deng et al., 2009) and a Long Short-Term Memory (LSTM) was used as decoder. In this work, we use ResNet152 (He et al., 2016) as the pretrained model for the encoder. The encoder-decoder architecture enables us to generate captions containing attributes such as: gender, style (here African/Western $)^{1}$, color, sleeve type and garment type.

\footnotetext{
${ }^{1}$ By African style we mean any outfit made of African fabric or inspired by traditional
} 
The other important contribution of this paper is that, unlike most of AI research works on fashion to the best of our knowledge, we are including African fashion images in the training set. We worked with a dataset containing both Western and African fashion images. Indeed, it is well known that the generalization capability of AI algorithms is intrinsically linked to the distribution of input data. Low diversified datasets lead to biases, and fashion AI is not an exception. It raises an ethical question as it could result in social exclusion with regards to access to AI technologies. It also results in an economic problem, as it limits the adoption of AI technologies by the African fashion industry for instance. And yet, the only sub-Saharan fashion market is worth $\$ 31$ billion according to the Textile Today magazine. This paper is the first one attempting to address this bias issue, using a dataset containing not only Western but African fashion images. The Western fashion dataset that we used here is a restricted version of DeepFashion, the one used by Zhu et al. (2017). The restricted DeepFashion datase ${ }^{2}$ is made of 78,979 images with attributes and captions. The African fashion dataset, named InFashAIv1, is from the Inclusive Fashion AI (InFashAI) project we are leading. InFashAIv1 is made of 15,716 item images, titles, prices and general descriptions. However, for the purpose of this paper we only used 8,842 images after some filtering procedures described further. Some annotation works have been done to standardize both datasets.

Our results showed that captioning quality is higher for Western fashion images as a consequence of this data gap. However, we showed that transfer learning occurs as using jointly both datasets increases African fashion image captioning quality significantly while preserving Western fashion image captioning quality. We exhibited it by comparing the joint learning to standalone learning.

The rest of the paper is structured in five (05) sections. Section 2 explores related work on fashion and ethics in AI. Section 3 introduces InFashAIv1 while section 4 presents the Show and tell model. Section 5 showcase our results and finally section 6 summarizes our main findings and contribution.

\section{Related work}

Fashion has been recently a major topic in Computer Vision (CV)(Donati et al., 2019; Xiao et al., 2017; Kiapour et al., 2014). Cheng et al. (2020) present a survey about more than 200 important studies related to fashion in the field of CV. It covers a large range of topics including fashion detection, fashion analysis, fashion synthesis and fashion recommendation. Here we focus on fashion detection and especially fashion image captioning. Image captioning draws lots of attention from the community. It generally involves attributes prediction. One striking evidence from the literature is that captioning is formalized in some studies as a multi-label classification problem (Chen et al., 2012; Yamaguchi et al., 2015; Chen et al., 2015; Sun et al., 2016) whereas others address the

African garments.

${ }^{2}$ We refer further to this restricted version just as DeepFashion. 
problem using an encoder-decoder model where a Convolutional Neural Network (CNN) is used to encode images and a Recurrent Neural Network (RNN), such as Long Short-Term Memory (LSTM), is used to decode a description containing desired attributes (Vinyals et al., 2015; Xu et al., 2015; Herdade et al., 2019; Yang et al., 2020).

Yang et al. (2020) are the first, at our knowledge, applying captioning to fashion images using the encoder-decoder structure. They suggest an improvement of state-of-the-art by introducing an Attribute-Level and Sentence-Level Semantic rewards as metrics to enhance generated descriptions relevance.

They also integrate attribute embedding, Reinforcement Learning (RL) in the description generation process and they built the FAshion CAptioning Dataset (FACAD). They achieved state-of-the-art performance compared to previously most known approaches. Nonetheless, their method relies on knowing the attributes of a fashion image to be able to get its description. If it could fit some needs in the fashion industry, it is at some point limited and cases where only images are available are more common.

In this paper we used the frame established by Vinyals et al. (2015). Peng et al. (2019) provide a robust Pythorch (Paszke et al., 2019) implementation of Vinyals et al. (2015)'s model that we use in this paper. Vinyals et al. (2015) showed by their experiment on the MSCOCO 2014 dataset (Lin et al., 2014a) that the attention mechanism introduced by Xu et al. (2015) enables caption generation that makes more sense but diminish the generalization ability of the model. Consequently we did not use the attention mechanism as we are mostly interested in attribute detection.

Ethics in Artificial Intelligence (AI) technologies is a hot topic today. Ethics in $\mathrm{AI}$ can be declined in several aspects but one of the most important is inclusiveness as it directly affects the real life performance of AI technologies. Chou et al. (2018) suggest that he most critical step in creating inclusive AI is to recognize where and how bias infects the system. An important dimension of inclusiveness is the representativeness of datasets used to learn a given task. A face detection algorithm would poorly perform on detecting people with a skin color not represented in the training set. The same concerns apply to fashion AI technologies. Indeed, fashion AI made significant progress in the last decade, at least partially, thanks to big datasets provided to the community such as: Fashion-mnist, Deepfashion, Fashion Landmark Dataset (FLD), Unconstrained Fashion Landmark Database and DeepFashion2 (Xiao et al., 2017; Liu et al., 2016a,b; Yan et al., 2017; Ge et al., 2019). Unfortunately, those datasets contain almost exclusively Western type clothing. This fact limits the extent in which they can be used, especially when it comes to processing fashion images of non-Western styles. Another concern is lack of diversity with regards to skin color of people in the images, the first type of bias raised by Chou et al. (2018). One major cause of this lack of diversity in fashion datasets is very likely the gap between the abundance of Western fashion platforms suitable for web scraping and on the other side the relative scarcity of similar platforms for African fashion. Still, the lack diversity could deepen not only social but economic inequalities. Indeed, the African fashion industry could unfortunately not benefit 
from significant advances offered by AIs trained on under-diversified datasets.

For all these reasons, we created the Inclusive Fachion AI (InFashAI) project aiming to build more diversified fashion datasets that would be shared with the scientific community.

\section{Towards more diversity in fashion datasets}

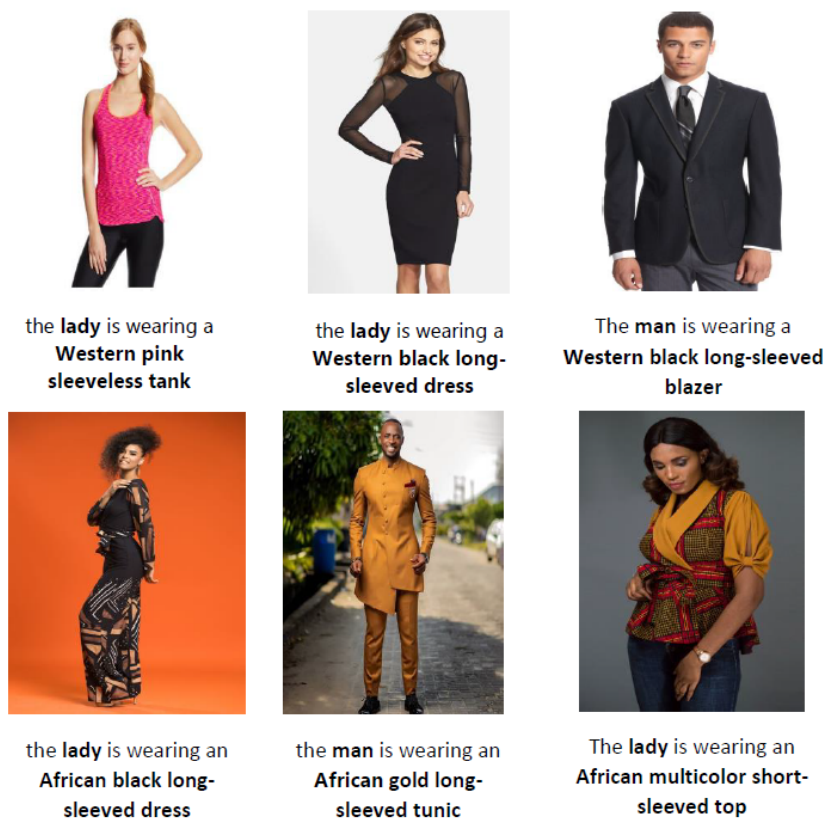

Figure 1: Some images with standardized descriptions from InFashAIv1

The first step of the InFashAI project was gathering data about African fashion. Our major sources of data are Pinterest and Afrikrea ${ }^{3}$. We collected 15,716 item images with their titles, prices (if provided) and general descriptions (if provided). This dataset named InFashAIv1 is available on Github.

In this paper, we used a restricted version of InFashAIv1 ${ }^{4}$. A first selection was made on images with the goal to only keep those depicting only one person. Also, to facilitate the learning task, images where further pre-processed in order to remove their backgrounds. We ended up with 8,842 images. For the purpose of this paper, we generated standardized captions with the following template: the $<$ gender $>$ is wearing a/an $<$ style $><$ color $><$ sleeve type $>$

\footnotetext{
${ }^{3}$ Afrikrea is one of the most known online market places specialized in African fashion, arts and crafts. We have a collaboration with them on the InFashAI project.

${ }^{4}$ We refer further to this restricted version simply as InFashAI
} 
$<$ garment type $>$. Detailed examples can be seen in Figure 1.

To generate standardized captions, we needed to annotate images attributes. We built a small mobile application with Appsheet, and we called for volunteers to help us with this task. Our annotated dataset is made of 8,842 images, with their standardized captions. In this work we use a concatenation of this annotated dataset (InFashAI) with the restricted DeepFashion dataset used by Zhu et al. (2017). This latter dataset is made of 78,979 images with their attributes and captions. We regenerated standardized captions for DeepFashion images using the template presented above. This allows us to have a larger dataset and should lead to better results on African fashion image captioning. Details on attributes distributions can be found in the appendix (Figure 2)

\section{Model architecture}

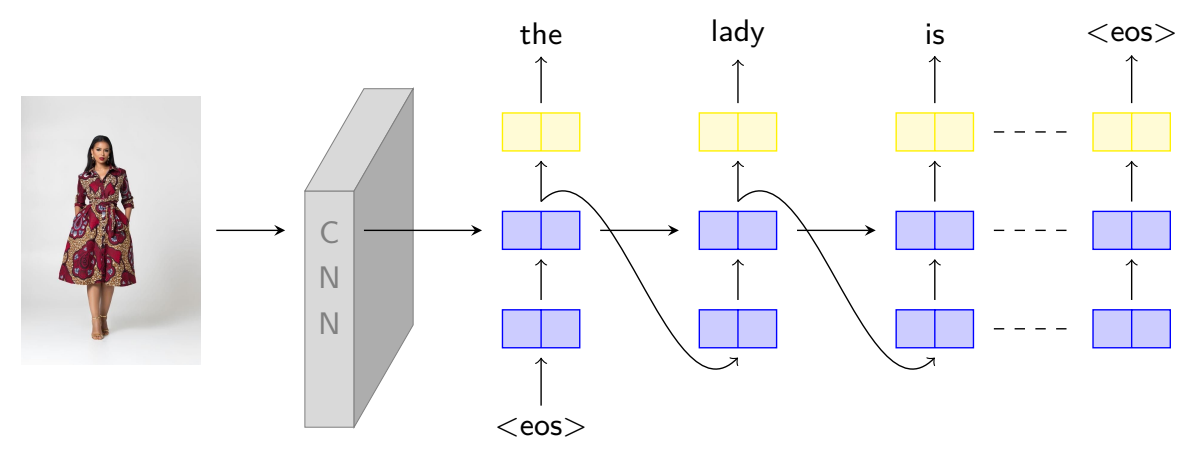

We are using here the model introduced by Vinyals et al. (2015) made of encoder-decoder architecture to generate novel descriptions from images. The encoder is a deep Convolutional Neural Network (CNN) used to encode an image $I$, projecting it in a textual embedding space. The decoder takes as initial input the projection of image $I$ throughout the encoder and its output is a sequence of $N$ words $S=\left\{S_{1}, S_{2}, \ldots, S_{N}\right\}$ that should be generated through a Recurrent Neural Network (RNN). The encoder-decoder is trained by maximizing the likelihood $p(S \mid I)$ :

$$
\theta^{*}=\arg \max \sum_{(I, S) \in D} \log p(S \mid I, \theta),
$$

where $\theta$ are the parameters of the model, $(I, S)$ is an image-caption pair, $D$ is the training sample. The joint probability over $S$ is defined as follows:

$$
\log p(S \mid I, \theta)=\sum_{t=1}^{N} \log p\left(S_{t} \mid I, \theta, S_{0}, \ldots, S_{t-1}\right),
$$

where $S_{t}$ is the $t^{t h}$ word in caption $S$ and $N$ the caption length. Vinyals et al. (2015) model the joint probability $p\left(S_{t} \mid I, \theta, S_{0}, \ldots, S_{t-1}\right)$ using Long-Short 
Term Memory (LSTM), a particular form of RNN:

$$
h_{t+1}=f\left(h_{t}, x_{t}\right),
$$

where $h_{t}$ is the $t^{t h}$ hidden layer, $x_{t}$ the input and $f$ is an activation function. To initialize the LSTM, the encoder output is used as $h_{0}$ and a start word as $x_{0}$. To generate the $t^{t h}$ word, previous output $S_{t-1}$ is used as input. After getting the $t^{t h}$ hidden layer, a softmax function is used to get a the conditional probability distribution over the vocabulary set for the next word prediction: $p_{t+1}=\operatorname{Softmax}\left(h_{t}\right)$.

\section{Results}

We used ResNet152 (He et al., 2016) as pretrained model for the encoder. We tested several set of parameters to find the one that would allow a transfer learning from Western fashion data. As the latter are more abundant, we wanted an architecture leveraging Western style captioning to improve African style captioning.

Here is the decoder's parameters we finally used:

- number of layers: 3

- hidden size: 512

- batch size: 50

- dropout: 0.3

- learning rate: 0.0002

- embedding size: 512

- number of epochs: 5

We cropped every images to size $224 \times 224$.

To limit noises from InFashAI images backgrounds, we used the tool developed by Nikita Selin to remove them. Most images from DeepFashion were already without any disturbing backgrounds. During our experiments, we realized that using a small batch size eases the learning of under-represented features.

African and Western images were concatenated to form a single pool. This pool contains 87,827 images. The common pool of images was subdivided into two parts: one for training ( $90 \%$ of the dataset, that is 79,044 images) and the rest for testing (8,783 images). Both training and test data contain African and Western images;

The training data is subdivided in 4 ways to obtain different training settings:

1. Train African: the training set only contains African images ( size : 7,802 images), 


\begin{tabular}{lrrr}
\hline & Test All & Test African & Test Western \\
\hline Train African & 0.376 & 0.474 & 0.365 \\
Train Western & 0.633 & 0.356 & 0.664 \\
Train Western $+1 / 2$ African & 0.650 & 0.526 & 0.664 \\
Train All & 0.650 & 0.530 & 0.663 \\
\hline
\end{tabular}

Table 1: BLEU-scores for different configurations

2. Train Western: the training set only contains Western images ( size: 70,287 images),

3. Train Western $+1 / 2$ African: the training set contains Western images and half of African images from Train African ( size: 74,188 images),

4. Train All: the training set contains Train Western and Train African ( size : 78,089 images).

In each training setting, a part or the complete training data is used to train the model. This leads to 4 different versions of the model where the difference only comes from the training data. Hyper-parameters used to train the model are identical for all settings. Each version of the model is evaluated on the complete test set (Test All: 8,783 images). Results are also reported respectively for African and Western data (Test African and Test Western).

Table 1 shows results for the BLEU scores for configurations we considered. When the model is trained on only one style (whether African or Western), it performs unsurprisingly well in terms of BLEU score on the test set of the same style: that is when trained on Train African (Western), the model performs better on Test African (Western): 0.474 VS 0.365 (0.356 VS 0.664).

When the model is trained on Train Western $+\mathbf{1} / \mathbf{2}$ African, we noticed a huge improvement of the BLEU score on Test African (0.526) while the BLEU score on Test Western remains stable.

When the model is trained on the full training set (Train All), results are very closed to the ones obtained with Train Western $+1 / 2$ African.

These results have serious implications when it comes to the amount of data needed to obtained reasonable results on diversified data. Indeed, in our case here, using only half of InFashAIv1 in addition to DeepFashion significantly improves results on African style while preserving results quality for Western style.

Nonetheless, the BLEU score is not enough to well capture the performance of the model. The vocabulary is limited to 69 words, and sentences were generated based on attributes. So, to get more accurate assessment of the model in the different settings, we computed F1 scores for each attribute. More precisely, we computed the weighted F1 score as implemented in the Scikit-Learn package to account for the weight of each modality (Pedregosa et al., 2011).

Table 2 shows F1 scores for every configurations we considered. The trend observed on the model performance based on the BLUE score seems also to 
Gender

\begin{tabular}{|c|c|c|c|}
\hline & Test All & Test African & Test Western \\
\hline Train African & 0.816 & 0.911 & 0.817 \\
\hline Train Western & 0.972 & 0.848 & 0.986 \\
\hline Train Western $+1 / 2$ African & 0.978 & 0.910 & 0.986 \\
\hline Train All & 0.976 & 0.912 & 0.984 \\
\hline \multicolumn{4}{|c|}{ Style } \\
\hline & Test All & Test African & Test Western \\
\hline Train African & - & - & - \\
\hline Train Western & - & - & - \\
\hline Train Western $+1 / 2$ African & 0.997 & 0.994 & 0.999 \\
\hline Train All & 0.997 & 0.994 & 0.999 \\
\hline \multicolumn{4}{|c|}{ Color } \\
\hline & Test All & Test African & Test Western \\
\hline Train African & 0.140 & 0.072 & 0.148 \\
\hline Train Western & 0.661 & 0.229 & 0.717 \\
\hline Train Western $+1 / 2$ African & 0.678 & 0.287 & 0.725 \\
\hline Train All & 0.685 & 0.296 & 0.732 \\
\hline \multicolumn{4}{|c|}{ Sleeve } \\
\hline & Test All & Test African & Test Western \\
\hline Train African & 0.312 & 0.492 & 0.293 \\
\hline Train Western & 0.841 & 0.635 & 0.864 \\
\hline Train Western $+1 / 2$ African & 0.839 & 0.695 & 0.855 \\
\hline Train All & 0.823 & 0.689 & 0.838 \\
\hline \multicolumn{4}{|c|}{ Garment type } \\
\hline & Test All & Test African & Test Western \\
\hline Train African & 0.179 & 0.211 & 0.176 \\
\hline Train Western & 0.517 & 0.199 & 0.559 \\
\hline Train Western $+1 / 2$ African & 0.539 & 0.338 & 0.561 \\
\hline Train All & 0.533 & 0.354 & 0.552 \\
\hline
\end{tabular}

Table 2: F1 scores for different configurations

Note: Train All: Trained on the concatenation of InFashAI and DeepFashion. 
apply to its ability to detect outfits attributes (gender, style, color, sleeve type and garment type). The F1 score is used to evaluate this attribute detection capacity.

The model trained on data from both clothing styles (Train All) gives quite high F1 scores for color detection when tested on Western clothing (Test Western): 0.732 against 0.717 if the model were only trained on Western data (Train Western). The F1 score obtained on Test African with the Train All model is 0.296 whereas the one obtained with the Train African and Train Western models are respectively 0.072 and 0.229 .

The performance gain of the model due to the training data diversity is also observed when detecting sleeve types, and particularly for African outfits. However, there is a slight deterioration in the performance of the model in the detection of this attribute when we jump from the Train Western $+\mathbf{1 / 2}$ African model to the Train All model. This could partially be due to some noises from errors in African images annotation.

We also noticed an improvement of the model in the detection of garment types, due to the diversity of the training data. Even if a slight deterioration on Test Western is observed when jumping from the Train Western $+\mathbf{1 / 2}$ African model to the Train All model, overall the performance gain due to the training data diversity largely outweighs this noise effect.

Additionally, as the model project images and captions in a common embedding space, we believe that the trained model could be used to generate image embedding and word embedding for tasks involving images and texts such as: image generation from text or image recommendation from text. Future works will showcase it.

\section{Conclusion}

This paper is one of the first tackling neural image captioning in the fashion domain. To build more inclusive fashion AI technologies, we created InFashAIv1, a dataset made of almost 16,000 African style fashion images with their titles, prices and general descriptions.

After some filtering procedures, we finally used almost 9,000 images from InFashAIv1 for our purpose in this paper. We concatenated this dataset with a restricted version of the DeepFashion dataset containing almost 79,000 images. In this work, we created standardized captions containing main attributes from images: gender, style, color, sleeve type and garment type.

We used the Show and Tell model with CNN encoder and RNN decoder to generate captions from images. Generated captions are satisfying with regards to feature detection especially on Western style images. However, the model trained on the augmented dataset showed significant improvement on attributes detection for African images compared to a model trained only on InFashAIv1, suggesting a transfer learning from Western style data (image-caption pairs).

We believe that the trained model can provide embedding for fashion images that encapsulates their main attributes. This embedding may be used for 
different tasks such as building an image retrieval system for fashion images.

A major caveat is that results from our model could be biased towards some stereotypes, like gender stereotypes. Potential biases are not treated in this paper and should be the object of further works.

Our work is a first step toward building more inclusive fashion AI tools. Though InFashAIv1 is an innovative dataset, its size is rather limited to get very reliable results and production-ready tools. We are working on extending this dataset to leverage broad research work.

The walk toward inclusive AI has long been stymied by the lack of suitable and large enough datasets. We believe that under-diversified datasets, such as DeepFashion, can be used in combination with small datasets, such as InFashAIv1, to leverage transfer learning and empower less biased and more generalizable models.

InFashAIv1 is released on Github to facilitate future works from the community that, we hope, would integrate more inclusiveness.

\section{Acknowledgements}

We are grateful to the Masakhane community and particularly to Julia Kreutzer for her constructive comments. We also thank Bonaventure Dossou and Chris Emezue for their helpful feedback. And we deeply thank all the volunteers who helped us to annotate African images.

\section{References}

Chen, H., Gallagher, A., and Girod, B. (2012). Describing clothing by semantic attributes. In European conference on computer vision, pages 609-623. Springer.

Chen, Q., Huang, J., Feris, R., Brown, L. M., Dong, J., and Yan, S. (2015). Deep domain adaptation for describing people based on fine-grained clothing attributes. In Proceedings of the IEEE Conference on Computer Vision and Pattern Recognition (CVPR).

Cheng, W.-H., Song, S., Chen, C.-Y., Hidayati, S. C., and Liu, J. (2020). Fashion meets computer vision: A survey. arXiv preprint arXiv:2003.13988.

Chou, J., Ibars, R., and Murillo, O. (2018). In pursuit of inclusive ai. Inclusive Design.

Deng, J., Dong, W., Socher, R., Li, L.-J., Li, K., and Fei-Fei, L. (2009). Imagenet: A large-scale hierarchical image database. In 2009 IEEE conference on computer vision and pattern recognition, pages 248-255. Ieee.

Donati, L., Iotti, E., Mordonini, G., and Prati, A. (2019). Fashion product classification through deep learning and computer vision. Applied Sciences, $9(7): 1385$. 
Everingham, M., Gool, L., Williams, C. K., Winn, J., and Zisserman, A. (2009). The pascal visual object classes (voc) challenge. International Journal of Computer Vision, 88:303-338.

Ge, Y., Zhang, R., Wang, X., Tang, X., and Luo, P. (2019). Deepfashion2: A versatile benchmark for detection, pose estimation, segmentation and reidentification of clothing images. In Proceedings of the IEEE/CVF Conference on Computer Vision and Pattern Recognition, pages 5337-5345.

He, K., Zhang, X., Ren, S., and Sun, J. (2016). Deep residual learning for image recognition. In Proceedings of the IEEE conference on computer vision and pattern recognition, pages $770-778$.

Herdade, S., Kappeler, A., Boakye, K., and Soares, J. (2019). Image captioning: Transforming objects into words. arXiv preprint arXiv:1906.05963.

Kiapour, M. H., Yamaguchi, K., Berg, A. C., and Berg, T. L. (2014). Hipster wars: Discovering elements of fashion styles. In European conference on computer vision, pages 472-488. Springer.

Lin, T.-Y., Maire, M., Belongie, S., Hays, J., Perona, P., Ramanan, D., Dollár, P., and Zitnick, C. L. (2014a). Microsoft coco: Common objects in context. In European conference on computer vision, pages 740-755. Springer.

Lin, T.-Y., Maire, M., Belongie, S. J., Hays, J., Perona, P., Ramanan, D., Dollár, P., and Zitnick, C. L. (2014b). Microsoft coco: Common objects in context. In $E C C V$.

Liu, Z., Luo, P., Qiu, S., Wang, X., and Tang, X. (2016a). Deepfashion: Powering robust clothes recognition and retrieval with rich annotations. In Proceedings of the IEEE conference on computer vision and pattern recognition, pages 1096-1104.

Liu, Z., Yan, S., Luo, P., Wang, X., and Tang, X. (2016b). Fashion landmark detection in the wild. In European Conference on Computer Vision, pages 229-245. Springer.

Paszke, A., Gross, S., Massa, F., Lerer, A., Bradbury, J., Chanan, G., Killeen, T., Lin, Z., Gimelshein, N., Antiga, L., Desmaison, A., Kopf, A., Yang, E., DeVito, Z., Raison, M., Tejani, A., Chilamkurthy, S., Steiner, B., Fang, L., Bai, J., and Chintala, S. (2019). Pytorch: An imperative style, highperformance deep learning library. In Wallach, H., Larochelle, H., Beygelzimer, A., d'Alché-Buc, F., Fox, E., and Garnett, R., editors, Advances in Neural Information Processing Systems 32, pages 8024-8035. Curran Associates, Inc.

Pedregosa, F., Varoquaux, G., Gramfort, A., Michel, V., Thirion, B., Grisel, O., Blondel, M., Prettenhofer, P., Weiss, R., Dubourg, V., Vanderplas, J., Passos, A., Cournapeau, D., Brucher, M., Perrot, M., and Duchesnay, E. (2011). 
Scikit-learn: Machine learning in Python. Journal of Machine Learning Research, 12:2825-2830.

Peng, Z., Dai, Y., Tang, Q., Cui, X., and Guo, S. (2019). Show and tell: A neural image caption generator.

Srinivasan, L. and Sreekanthan, D. (2018). Image captioning-a deep learning approach.

Sun, G.-L., Wu, X., and Peng, Q. (2016). Part-based clothing image annotation by visual neighbor retrieval. Neurocomputing, 213:115 - 124. Binary Representation Learning in Computer Vision.

Vinyals, O., Toshev, A., Bengio, S., and Erhan, D. (2015). Show and tell: A neural image caption generator. In 2015 IEEE Conference on Computer Vision and Pattern Recognition (CVPR), pages 3156-3164.

Wang, H., Zhang, Y., and Yu, X. (2020). An overview of image caption generation methods. Computational Intelligence and Neuroscience, 2020.

Xiao, H., Rasul, K., and Vollgraf, R. (2017). Fashion-mnist: a novel image dataset for benchmarking machine learning algorithms. arXiv preprint arXiv:1708.07747.

Xu, K., Ba, J., Kiros, R., Cho, K., Courville, A., Salakhudinov, R., Zemel, R., and Bengio, Y. (2015). Show, attend and tell: Neural image caption generation with visual attention. In International conference on machine learning, pages 2048-2057. PMLR.

Yamaguchi, K., Okatani, T., Sudo, K., Murasaki, K., and Taniguchi, Y. (2015). Mix and match: Joint model for clothing and attribute recognition. In $B M V C$, volume 1 , page 4 .

Yan, S., Liu, Z., Luo, P., Qiu, S., Wang, X., and Tang, X. (2017). Unconstrained fashion landmark detection via hierarchical recurrent transformer networks. In Proceedings of the 25th ACM international conference on Multimedia, pages $172-180$.

Yang, X., Zhang, H., Jin, D., Liu, Y., Wu, C.-H., Tan, J., Xie, D., Wang, J., and Wang, X. (2020). Fashion captioning: Towards generating accurate descriptions with semantic rewards. arXiv preprint arXiv:2008.02693.

Zhu, S., Fidler, S., Urtasun, R., Lin, D., and Chen, C. L. (2017). Be your own prada: Fashion synthesis with structural coherence. In Proceedings of the IEEE Conference on International Conference on Computer Vision. 


\section{Appendix}

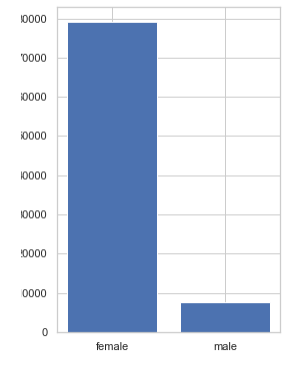

(a) Gender

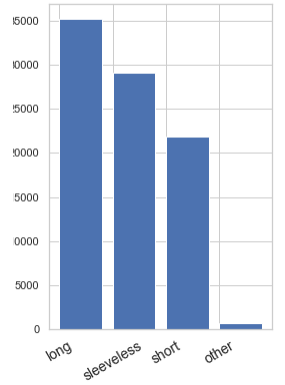

(b) Sleeve type

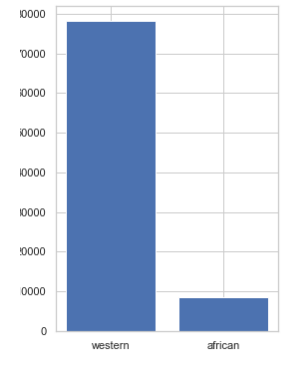

(c) Style

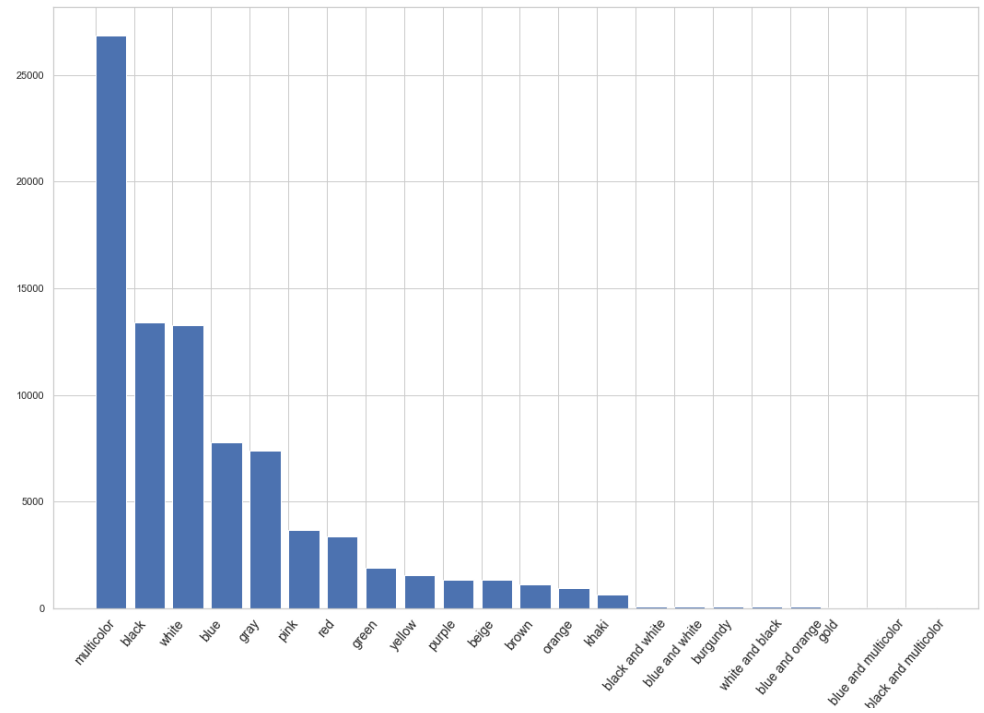

(d) Color

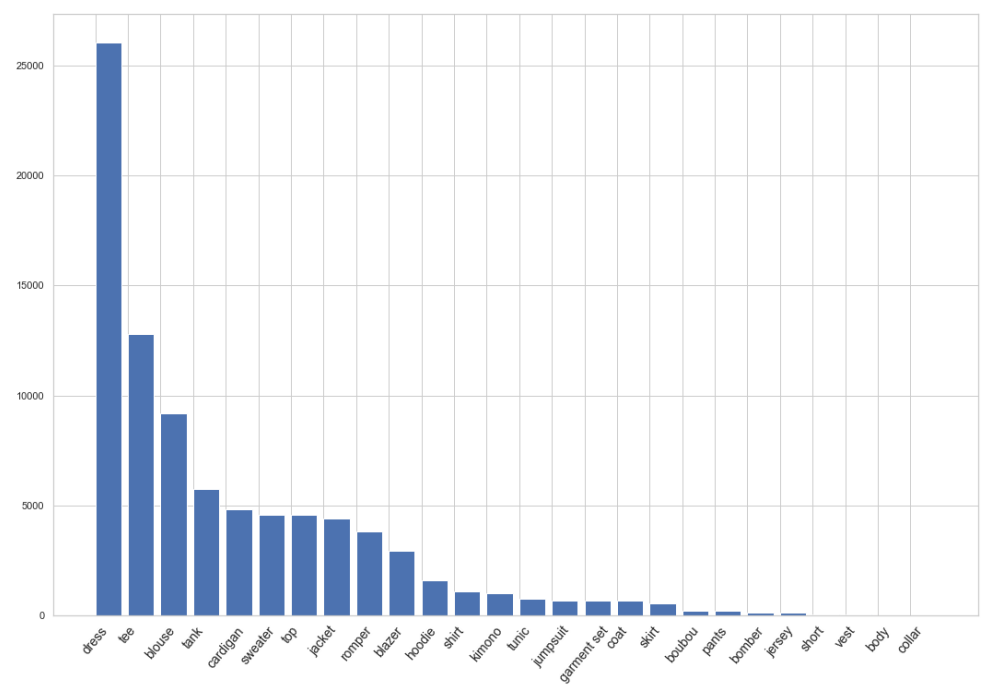

(e) Garment type 